
\title{
ZS Resarach Square \\ Performance Evolution of Modern Error Controlling Codes for future generation (5G) cellular systems: LDPC code
}

\section{Pradeep M Shah ( $\sim$ pradeepmshah@yahoo.co.in )}

Anand Engineering College, GCET https://orcid.org/0000-0003-4370-609X

\section{Prakash D Vyavahare}

Shri Govindram Seksaria Institute of Technology and Science

\section{Anjana Jain}

Shri Govindram Seksaria Institute of Technology and Science

\section{Research}

Keywords: LDPC: Low Density Parity Check Codes, EXIT Chart: Extrinsic Information Chart, 5G: Fifth Mobile Generation Network: Worldwide Interoperability for Microwave Access, FDMA: Frequency Division Multiple Access, OFDM: Orthogonal Frequency Division Multiplexing, CDMA: Code Division Multiple Access, UMTS: Universal Mobile Telecommunication

Posted Date: May 22nd, 2020

DOl: https://doi.org/10.21203/rs.3.rs-22526/v1

License: (c) (1) This work is licensed under a Creative Commons Attribution 4.0 International License. Read Full License 


\section{Abstract}

Modern error controlling codes, like a Low-Density Parity Check (LDPC) codes, assume a significant job in a next-generation wireless system like $5 \mathrm{G}$ for improved channel error controlling ability. Execution of Turbo codes(3G), Polar codes(4G) and LDPC (5G) codes are near to Shannon's limit channel codes and in this manner are being utilized in next-generation wireless communication systems. This paper emphasis around modern cellular cell network, LDPC codes and their performance using EXIT chart is presented. Likewise, a similar exhibition assessment of LDPC codes and the decisions of error controlling codes for the future generation cell systems are talked about in the paper. Such an investigation will be helpful in the choice of legitimate LDPC codes for the next-generation wireless communication system.

\section{Introduction}

In wireless communication systems, received data bits normally adjust to the transmitted bits due to the blunders happened by an obstruction, poor sign quality. To address these data bits, wireless cellular communication systems use error control codes. In the last two decades, there has been a significant increase in research for new cellular technologies because of the convenience and need for higher data rate access. Present day correspondence framework is required to help expanded information rates, consistent client access to the spine of communication network and incorporate various services, for example, downloading and transferring motion pictures and recordings, video conferencing, observation for security reason, tele medication and tele mechanical technology. From $1 \mathrm{G}$ to $5 \mathrm{G}$, this universe of media transmission has seen various enhancements along with improved execution as time passes. Table 1 shows a different generation (1G to5G) [1] in Mobile communication systems and their important features.

Table I. Performance of Cellular Systems from 1G-5G

\begin{tabular}{|c|c|c|c|c|c|}
\hline & $1 \mathrm{G}$ & $2 G$ & $3 G$ & $4 G$ & $5 \mathrm{G}$ \\
\hline Freq. & $824-894$ & $850-1900$ & $1.5-2.8$ & $2-8$ & $3-300$ \\
\hline Band & $\mathrm{MHz}$ & $\mathrm{MHz}$ & GHz & $\mathrm{GHz}$ & $\mathrm{GHz}$ \\
\hline $\begin{array}{l}\text { Band- } \\
\text { Width }\end{array}$ & $30 \mathrm{KHz}$ & $\begin{array}{l}1.25 \\
\mathrm{MHz}\end{array}$ & $5 \mathrm{MHz}$ & $\begin{array}{l}20 \\
\mathrm{MHz}\end{array}$ & $100 \mathrm{MHz}$ \\
\hline $\begin{array}{l}\text { Data } \\
\text { Rate }\end{array}$ & $\overline{\text { Few kbps }}$ & $\begin{array}{l}22.8 \\
\text { Kbps }\end{array}$ & $\begin{array}{l}\text { Up to } \\
2 \mathrm{MHz}\end{array}$ & $\begin{array}{l}\text { Up to } \\
20 \mathrm{MHz}\end{array}$ & $\begin{array}{c}1 \text { Gbps or } \\
\text { high }\end{array}$ \\
\hline Access & FDMA & $\overline{\mathrm{CDMA}}$ & $\begin{array}{l}\text { WCD } \\
\text { MA }\end{array}$ & OFDM & $\begin{array}{l}\text { OFDM } \\
\text { MIMO }\end{array}$ \\
\hline $\begin{array}{l}\text { Appli- } \\
\text { Cation }\end{array}$ & $\begin{array}{l}\text { Analog } \\
\text { Cellular } \\
\text { Tech }\end{array}$ & $\begin{array}{c}\text { Digital } \\
\text { Voice } \\
\text { services }\end{array}$ & \begin{tabular}{|c|} 
Multi \\
media \\
services
\end{tabular} & $\begin{array}{l}\text { Voice } \\
\text { And } \\
\text { Data }\end{array}$ & $\begin{array}{l}\text { Very High } \\
\text { data rate }\end{array}$ \\
\hline $\begin{array}{l}\text { Deploy- } \\
\text { Ment }\end{array}$ & 1980's & 1990's & 2000's & 2010's & 2020's \\
\hline
\end{tabular}


The cutting-edge telecom systems (5G) will begin hitting the market in 2020 and will keep on growing around the world. With simply speed enhancements, $5 \mathrm{G}$ is relied upon to release an enormous loT biological system where systems can serve correspondence requirement for billions of associated gadgets, with the correct exchange offs between information rates, time - idleness and cost. In $5 \mathrm{G}$ we have to introduce the LDPC code instead of turbo code, which are utilized in Wi-Fi, Wi MAX, Gigabit ethernet and DVB-S2 guidelines [2], or with the moderately polar codes. With the stringent demands of 5G communications, the best among the current candidate codes are as follows:

- Turbo Codes (used in $4 \mathrm{G}$ already).

- Polar Codes (2008)

- LDPC Codes (used in 5G Better than Turbo)

The rest of the section of this paper are sorted out as pursues. Section II gives foundation data of LDPC codes. Section III furnishes the detail plan LDPC codes with the assistance of the EXIT diagram and in Section IV the exhibition correlations of different error controlling codes for cutting edge portable systems are talked about. At long last, conclusions are a notice in Section V.

\section{Related Works Of Proposed Code For 5g: Ldpc Codes}

\section{A. Basics of LDPC codes}

Low-Density Parity-Check Codes (LDPC) are special types of linear block codes which having huge codeword length. These LDPC codes were designed by Robert Gallager [3]. However, these codes were not generally utilized because of their high computing complexity nature. In 1996, Mackay and Neal [4] rethought LDPC codes, they demonstrate theirs close as far as possible execution LDPC codes have better error controlling execution as contrasted to Turbo codes with huge block length and lower decoding complexity nature. LDPC codes are represented by the accompanying subsequent important characteristics:

- Improved execution as contrasted to Turbo code

- Lower computational nature

- Higher adaptability because of parallel ability

- High throughput

With their exceptional highlights and execution, LDPC codes are utilized in various applications, for e.g., $5 \mathrm{G}[5]$.

\section{B. Matrix Representation of LDPC codes}

We can indicate LDPC codes as $\left(n, W_{c}, W_{r}\right)$, wherever $n$ is the code length, $W_{c}$ is the section weight and $W_{r}$ is the column weight. In standard LDPC codes, $W_{c}$ and $W_{r}$ stay invariant among entire rows and columns, 
whereas in irregular codes, $W_{c}$ and $W_{r}$ are vary among various rows and columns. All types of LDPC codes [6], must fulfil following equation

$$
H^{\top} \mathrm{X}=0
$$

wherever $\mathrm{X}$ is a code word and $\mathrm{H}$ is parity check matrix. A matrix is sparse if at least half the numbers of elements in its $\mathrm{H}$ matrix have zero value. The term low density refers to the low concentration of ones in the parity check matrix of LDPC codes. Let, there be $\mathrm{k}$ sources bits in every $\mathrm{n}$ - bits codeword. At that point, $\mathrm{H}$ is a matrix given by ( $\mathrm{n}-\mathrm{k})$ rows, each representing generation of one check bit and $\mathrm{n}$ columns. Here we take $(8,4)$ LDPC code, whose parity check matrix is

$$
H=\left[\begin{array}{llllllll}
0 & 1 & 0 & 1 & 1 & 0 & 0 & 1 \\
1 & 1 & 1 & 0 & 0 & 1 & 0 & 0 \\
0 & 0 & 1 & 0 & 0 & 1 & 1 & 1 \\
1 & 0 & 0 & 1 & 1 & 0 & 1 & 0
\end{array}\right]
$$

Note: For the code under consideration $\mathrm{W}_{\mathrm{c}}=2$ and $\mathrm{W}_{\mathrm{r}}=4$.

\section{Tanner Graph Representation of LDPC codes}

Tanner charts are called bipartite diagrams, which can be utilized to characterize LDPC codes. In Tanner charts, nodes characterize into two classes: check nodes and variable nodes, where variable node represents one column of the parity check matrix, and check nodes, represent one row of the parity check matrix. Figure 1 is a case of a bipartite Tanner chart for the LDPC code demonstrated to by parity check matrix represented in Eq. (2), in which $(\mathrm{C} 1, \mathrm{C} 2, \mathrm{C} 3, \mathrm{C} 4)$ represent row message bits and (V1, V2, V3... V8) represents column values (code word).

\section{Experimental Result \& Performance of LDPC code:}

For performance evolution, MATLAB simulation of LDPC code shown in Fig. 2. Here compares the performance of LDPC code with the different code rate. From the graphs we can conclude that codes with a short length are used for low or medium code rates and codes with a long length like LDPC are used for higher code rate. LDPC code are long code which lead to large latency (1000 bits or so) and required higher power consumption. Low latency, lower power consumption and short block length codes are very much in need. Improvement for short block length are still open.

\section{E. LDPC Prptograph}

A protograph is a Tanner graph which is repeat to make a bigger graph. Protograph [7] construction is way of constructing parity check matrix. First define the base matrix or photo matrix which is expanded to get the actual parity check. These expansions are done by right shift permutation matrices. For $\mathrm{i}^{\text {th }}$ generally small number of nodes, associated by few edges, permitting parallel edges between two nodes. 
A protograph is typically represented by a protomatrix, i.e., a matrix whose entries indicate the number of edges connecting the respective variable and check nodes. Therefore, unlike the parity check matrix, the protomatrix is in general non-binary. A simple example of a protograph is shown in Fig. 3.

This chart comprises of 3 variable nodes and 2 check hubs that are interrelated by 7 diverse edge (variable-to-check) types. The inferred diagram is developed by imitating the protograph multiple times and permuting variable-to-check pairings inside a similar edge kind of the protograph. For instance, the protomatrix of protograph in Fig. 2. is in the accompanying structure

$$
\mathrm{H}_{\text {proto }}=\left(\begin{array}{lll}
1 & 1 & 1 \\
2 & 1 & 1
\end{array}\right)
$$

where the lines and sections speak to the check nodes and variable nodes in the chart separately. The (i; j) component of the protomatrix shows the quantity of parallel edges that associate the check node $i$ and the variable node $\mathrm{j}$. The protograph code diagram has $\mathrm{N}$ times the same number of nodes as the protograph, however contrasted and the protograph it has a similar rate just as a similar degree appropriation for both variable and check nodes. Protograph LDPC codes are a subclass of multi-edge type LDPC codes where each edge of a protograph is one edge type. Protograph codes can accomplish limit moving toward execution with low encoder multifaceted nature just as quick disentangling [8]

\section{Result Discussion Using Exit Chart}

Extrinsic Information (EXIT) chart is a technique which allows obtaining near-optimal performance parameters for LDPC code [9]. Tanner diagram which has $\mathrm{m}$ check nodes and $\mathrm{n}$ variable nodes can be described by degree $d v(i)$ and $d c(j)$, where $d v(i)$ is the level of the $i^{\text {th }}$ variable node and $d c(j)$ is the level of $j^{\text {th }}$ check node.

An EXIT chart as appeared in Fig. 4 is for standard LDPC codes with $d v(i)=d v=8$ for $i=1,2 \ldots n$ and $d c(j)$ $=\mathrm{dc}=4 \mathrm{for} j=1,2 \ldots \mathrm{m}$. In the figure, the measurement utilized for transfer curve is extrinsic mutual information, therefore it is recognized as EXIT chart. As appeared in the figure, the decoding trajectory corresponds to these EXIT bends. As SNR is expanded, the top bend moved upward and expanding the passage width between the two bends and consequently the decoder convergence rate likewise expanded. Here SNR is simply over the decoding edge for code $(d v, d c)=(8,4)$ and $(E B / N 0)$ thes $=1.1 d B$.

\section{Performance Comparisions}

The selection of the suitable error controlling codes has a very important in the achievement of the future generation cellular systems. There is no universal coding method which is all-around used. The best suitable coding method is subject to the number of parameters, for example, code rate, BER, Block length, and decoding complexity nature, etc. In the accompanying Table II, the different error controlling codes 
for future generation cellular systems appear. From table, we can observe that the LDPC code play very important role for fifth generation (5G) mobile communication systems.

Table II. Comparison of different error controlling codes for future generation cellular systems.

\begin{tabular}{|llll|}
\hline & & \multicolumn{2}{l|}{ Error controlling codes } \\
\hline & RS, & & LDPC codes, \\
& BCH codes & Turbo Codes & Protograph \\
\hline Mobile & $1 G$ and $2 \mathrm{G}$ & $3 \mathrm{G}$ and $4 \mathrm{G}$ & LDPC codes \\
\hline Generations & & & \\
\hline Types & Cyclic & Convolutional beyond & Block \\
\hline Code Rate & $1 / 6,1 / 4$ & $1 / 3,{ }^{1 / 2}$ & $2 / 3,3 / 4,7 / 8$ \\
\hline Block length & Small & Small & Large \\
\hline Supported & GPRS & LTE & WiMAX, DVB- \\
\hline Standards & & & S2 \\
\hline BER & Poor & Better & Best \\
\hline Interleaver & No & Yes & No \\
\hline Used & & & \\
\hline Decoding & Moderate & Higher & Lower \\
\hline Complexity & & & \\
\hline
\end{tabular}

\section{Conclusion}

In this paper, we estimate a modern error controlling code, LDPC codes by EXIT chart method which is used to evaluate the performance of near Shannon limit channel codes. The analysis will be useful in the selection of a channel code for $5 \mathrm{G}$ system and above. Here, we have examined the LDPC codes, which can meet the necessities for future generation (5G), with very high data throughputs up to $20 \mathrm{Gbps}$, $\mathrm{EB} / \mathrm{NO}=1.1 \mathrm{~dB}$ and greater flexibility than the previous generation of mobile communication systems for meeting quality servicers requirement of various types of traffic including voice, video, and data.

\section{Abbreviations}

LDPC: Low Density Parity Check Codes, 
EXIT Chart: Extrinsic Information Chart,

5G: Fifth Mobile Generation Network

Wi-Max: Worldwide Interoperability for Microwave Access,

FDMA: Frequency Division Multiple Access,

OFDM: Orthogonal Frequency Division Multiplexing,

CDMA: Code Division Multiple Access,

UMTS: Universal Mobile Telecommunication.

\section{Declarations}

\section{Availability of data and materials}

We declared that materials described in the manuscript, including all relevant raw data, will be freely available to any scientist wishing to use them for non-commercial purposes, without breaching participant confidentiality.

\section{Funding}

Not applicable

\section{A. Acknowledgements}

The authors would like to thank all the referees for their constructive and insightful comments on this paper.

\section{B. Author information}

Contributions:

Pradeep M. Shah wrote the entire article. The author(s) read and approved the final manuscript.

\section{A. Corresponding authors}

Correspondence to Pradeep M. Shah 


\section{B. Ethics declarations}

\section{Ethics approval and consent to participate}

This article does not contain any studies with human participants or animals performed by any of the authors.

\section{Competing interests}

No competing interest.

\section{References}

1. Shah P. M., Vyavahare P.D., Jain Anjana, "Modern Error-Correcting Codes: Turbo and LDPC codes" IEEE RADIO-2015.

2. Zhang, H. Liu, X. Chen, D. Liu, and X. Yi, “Low complexity E. DVB- S2 LDPC decoder," in Proc. IEEE Veh. Technol. Conf., Barcelona, Spain, April 2009.Author, F.: Contribution title. In: 9th International Proceedings on Proceedings, pp. 1-2. Publisher, 2010.

3. GALLAGER, R.G. "Low- density parity check codes," IRE Trans Information Theory, IT-8, pp 21-28, 1962.

4. J.C. Mackay and R.M. Neal, "Near Shannon's limit performance of the low-density parity-check codes," Electron. Lett., vol.32, pp.1645-1646, Aug.1996.

5. G. Maunder, "A vision for 5G channel coding" a white paper, University of Southampton Dataset, Acceleron, 2016.

6. Mohsenin, D. N. Truong, and B. M. Baas, "A low-complexity message-passing algorithm for reduced routing congestion in LDPC decoders," IEEE Trans. Circuits Syst., vol. 57, no. 5, pp. 1048-1061, May 2010 .

7. G. Maunder, "A vision for 5G channel coding" a white paper, University of Southampton Dataset, Accelercomm, 2016.

8. T. Mohsenin, D. N. Truong, and B. Baas, "A low-complexity message-passing algorithm for reduced routing congestion in LDPC decoders," IEEE Trans. Circuits Syst. I, vol. 57, no. 5, pp. 1048-1061, May 2010.

9. Zhang, V. Anantharam, M. J. Wainwright, and B. Nikolic, "An efficient 10GBASE-T Ethernet LDPC decoder design with low error floors," IEEE J. Solid-State Circuits, vol. 45, no. 4, pp. 843-855, April 2010.

10. Gautham Prasad, Haniph A. Latchman, Low-density Lee, Weiler A. Finamore. "A Comparative Performance Study of LDPC and Turbo Codes for Realistic PLC Channels" Power Line Communication and its Application (ISPLC), 18 $8^{\text {th }}$ IEEE International Symposium, pp.,201-207,2014. 
Figures

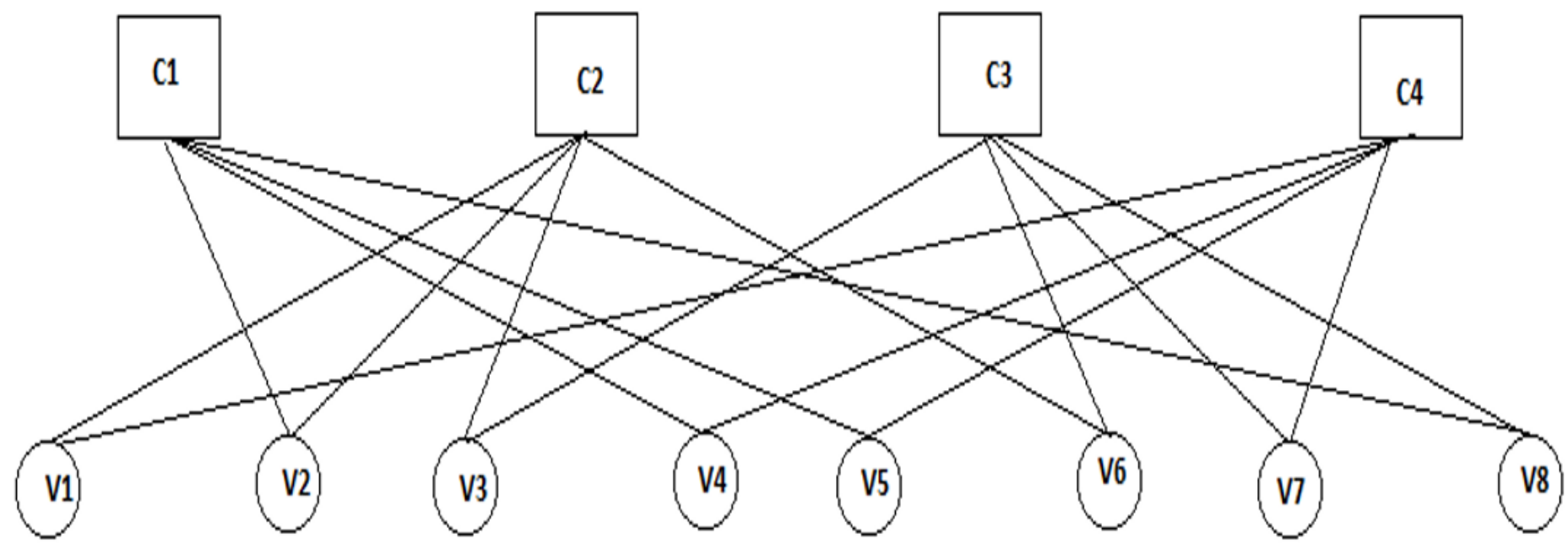

Figure 1

Tanner graph for $(8,4)$ LDPC code. 


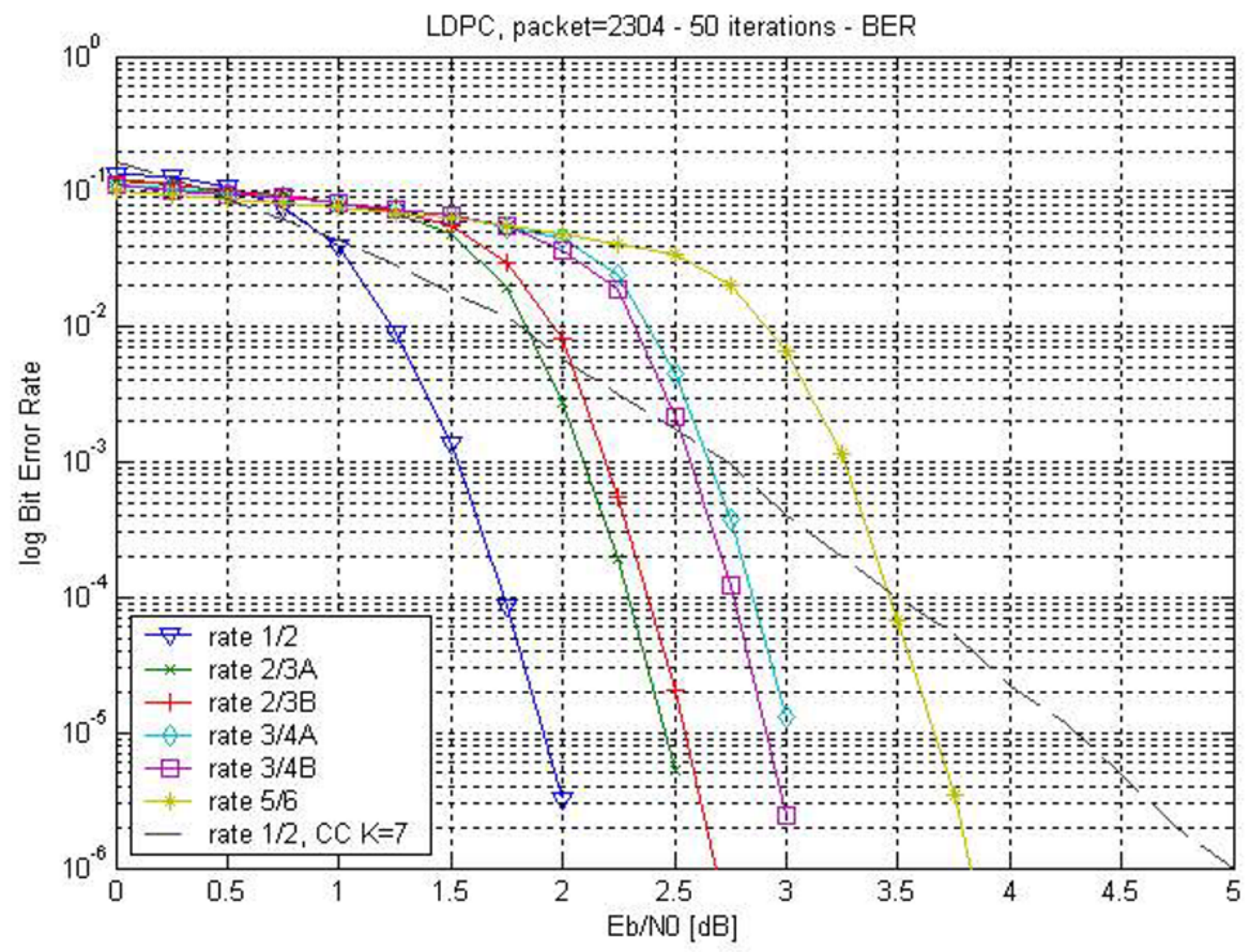

Figure 2

LDPC performance for different code rate. 

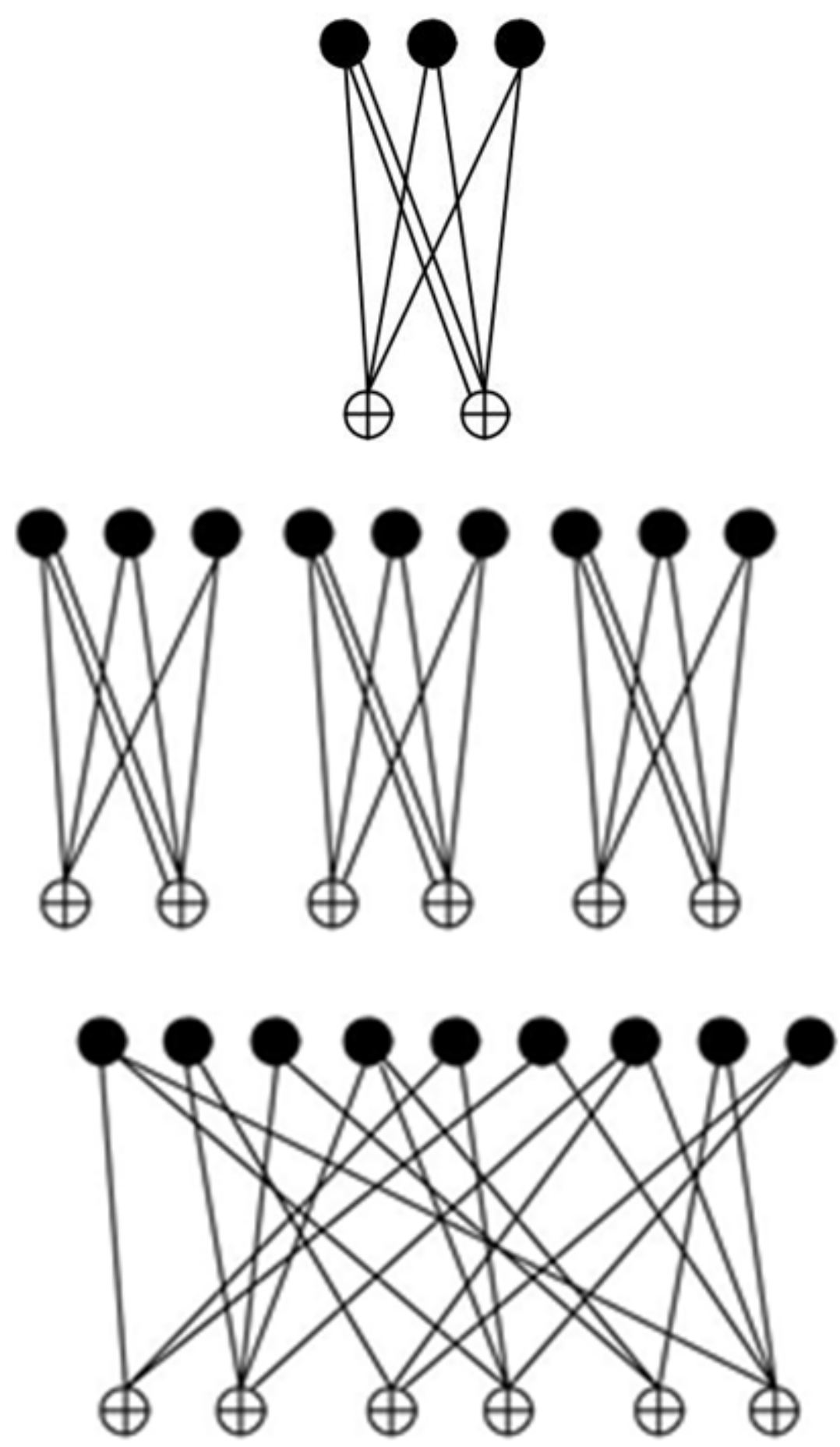

Figure 3

Copy and permute operation for a protograph 


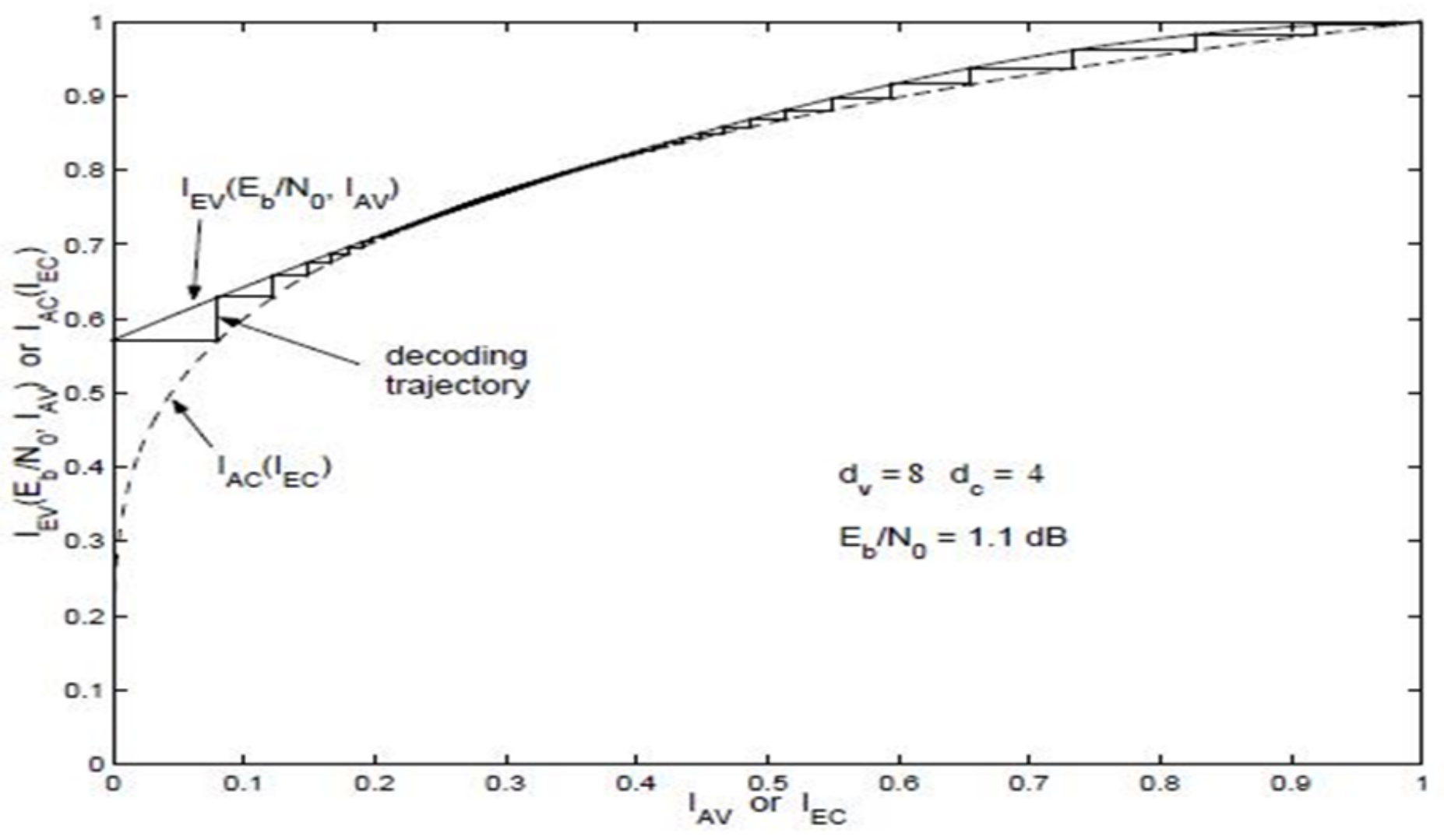

Figure 4

EXIT chart for regular LDPC code. 